\title{
Global nonlinear optimization for the estimation of static shift and interpretation of 1-D magnetotelluric sounding data
}

\author{
Shashi Prakash Sharma ${ }^{1, \star}$ and Arkoprovo Biswas ${ }^{1}$ \\ ${ }^{1}$ Indian Institute of Technology, Department of Geology and Geophysics, Kharagpur, India
}

\author{
Article history \\ Received June 21, 2010; accepted March 11, 2011. \\ Subject classification: \\ Magnetotelluric sounding, Global nonlinear optimization, Static shift, Transverse electric and magnetic modes, Apparent resistivity, Phase
}

\section{ABSTRACT}

In the presence of conducting inhomogeneities in near-surface structures, apparent resistivity data in magnetotelluric sounding can be severely distorted. This is due to electric fields generated from boundary charges on surficial inhomogeneities. Such distortion persists throughout the entire recording range and is known as static shift in magnetotellurics. Frequencyindependent static shifts manifest as vertical, parallel shifts that occur in plots of the dual logarithmic scale of apparent resistivity versus time period. The phase of magnetotelluric sounding data remains unaffected by the static shift and can be used to remove the static shift to some extent. However, individual inversion of phase data yields highly nonunique results, and alone it will not work to correctly remove the static shift. Inversions of uncorrected magnetotelluric data yield erroneous and unreliable estimations, while static-shift-corrected magnetotelluric data provide better and reliable estimations of the resistivities and thicknesses of subsurface structures. In the present study, static shift (a frequencyindependent real constant) is also considered as one of the model parameters and is optimized together with other model parameters (resistivity and thickness) using the very fast simulated annealing global inversion technique. This implies that model parameters are determined simultaneously with the estimate of the static shift in the data. Synthetic and noisy data generated for a number of models are interpreted, to demonstrate the efficacy of the approach to yield reliable estimates of subsurface structures when the apparent resistivity data are affected by static shift. Individual inversions of static-shift-affected apparent resistivity data and phase data yield unreliable estimations of the model parameters. Furthermore, the estimated model parameters after individual data inversions do not show any systematic correlations with the amount of static shift in the data. The present study shows that only joint inversion of the apparent resistivity and phase data, without or with optimizing of the static shift, yields models that show good fits between the observed and the model data. Joint inversion results also reveal a systematic relationship between the estimated model parameters and the static shift in the data. The proposed approach also shows that estimated resistivities are ' $\mathrm{S}$ ' (the static shift parameter) times the actual resistivities, and that estimated thicknesses are $\sqrt{ } \mathrm{S}$ times the actual thicknesses without optimization of the static shift. This result is in good agreement with the existing relationship in the literature. Therefore, the global optimization procedure developed can be effectively used to optimize the static shifts in data, to obtain reliable estimations of model parameters. Subsequently, joint inversion of the apparent resistivity and phase data, with optimization of the static shift, is performed, which yields accurate estimates of subsurface structures. It is demonstrated that this approach can also be used when the data is not affected by the static shift. In such cases, the estimated static shift parameter 'S' will be close to unity. The efficacy of the approach is demonstrated with a field example from Singhbhum craton, eastern India, by providing an accurate estimation of the craton thickness and the conducting structure that lies below the craton.

\section{Introduction}

The magnetotelluric method is an important exploration technique for investigation of deep resistivity structures within the Earth [e.g., Swift 1967, Vozoff 1972, Berdichevsky et al. 1980, Vanyan et al. 1983, Wannamaker et al. 1984]. The magnetotelluric method involves the measurement of various components of time-varying electric and magnetic fields. The presence of electric charges on local surficial, or near-surface, lateral inhomogeneities produces local electric fields, which in turn affect the actual electric field at a regional scale. The horizontal components of the electric field of the Earth that is of regional interest are perturbed due to the presence of spurious local electric fields, and hence in principle the disturbed electric field components can be measured. Such perturbations in the electric field cause a parallel shift of the apparent resistivity curve in the dual logarithmic scales of the plot of the apparent resistivity versus frequency (or time period), and this is known as static shift (multiplication by a frequencyindependent real constant) [Groom and Bailey 1989, Cerv et al. 2010]. If the static shift is not identified and not corrected in the data, then inversion/interpretation of these data yields incorrect estimates of the resistivity and thickness of subsurface structures. Inversion of magnetotelluric sounding 
data without the removal of the static shift means that the interpreted resistivities are multiplied by a factor equal to the amount of the parallel shift between the apparent resistivity curves, and the interpreted thicknesses are multiplied by an amount related to the square root of this shift in the inverted resistivity [e.g., Sternberg et al. 1988]. Therefore, the static shift should be removed with greater accuracy for more reliable interpretations of magnetotelluric sounding data.

Sternberg et al. [1988] have reviewed a number of methods to overcome this static shift problem, such as: (a) spatial filtering using closely spaced magnetotelluric sites; (b) theoretical calculations of the static shift from buried surface inhomogeneities; (c) theoretical calculations of the static shift from surface topographic effects; (d) interpretation based on known geology; and (e) use of independent measurements. The use of transient electromagnetic sounding together with magnetotelluric sounding for removal of the static shift was described by Sternberg et al. [1988]. As the static shift is caused by boundary charges that mainly affect the electric field, measurement of magnetic fields will overcome this problem. The apparent resistivity of the Earth can also be determined from a single measurement of the magnetic field, using a controlled-source method. Sternberg et al. [1988] used the Geonics EM37 time-domain recording system to make central-loop induction soundings. They showed that transient electromagnetic soundings can be compared to magnetotelluric data by shifting the time scale of the transient electromagnetic soundings by a factor, and hence they estimated the static shift in magnetotelluric data.

Jones [1988] applied a parameter constraint method to remove the static shift from magnetotelluric sounding data. This method proposed by Jones [1988] assumes that one of the layers in the sequence, say the $i^{\text {th }}$ layer, can be represented in a parametric fashion, and estimations of the resistivity given by $\rho_{i}(x, y)$ at any point $(x, y)$ vary about the true value in a statistical manner. de Groot-Hedlin [1991] presented a regularized inversion for the removal of static shift, where a method for jointly handling the static shift and the model parameters was discussed. Various other studies that have dealt with static-shift removal have been discussed in the literature [e. g., Larsen 1977, Pellerin and Hohmann 1990, Beamish and Travassos 1992, Wang et al. 1997, Macnae et al. 1998]. Bahr [1991] also reviewed a number of approaches used to remove the static shift from magnetotelluric sounding data in various studies.

As shown by Sternberg et al. [1988] and de Groot-Hedlin [1991], the optimization of the static shift with the other model parameters can solve the problem of static shift and yield reliable model parameters. Since solutions using linearized inversion converge in the vicinity of the initial guesses, optimization of the static shift along with the other model parameters using linearized inversion will yield a model that fits the observed data for any value of static shift. Hence, estimation of model parameters using linearised inversions might not be reliable. As any value of static shift can fit the data equally well, this can still give inaccurate estimations of resistivity and thickness.

Global optimization methods that are free from bias of the initial model might represent the correct approach to finding the correct value of the static shift, as compared to linearized inversion. Therefore, in the present study, the static shift is considered as one of the model parameters and it is optimized along with the other model parameters (resistivities and thicknesses) using very fast simulated annealing (VFSA) global optimization. As global inversion yields a number of solutions in a predefined model space, to obtain a more accurate solution, a statistical mean model is computed from all of the solutions. A number of models depicting different geological conditions are studied. The efficacy of the approach is also demonstrated with the help of field data, as this will yield better results than linearized inversion approaches.

\section{Theoretical background}

\subsection{Magnetotelluric sounding}

Spurious electric fields caused by near-surface inhomogeneities affect actual electric fields generated by the electromagnetic induction phenomena in the Earth subsurface. The apparent resistivities at various time periods are affected by a multiplicative error (multiplication by a frequency-independent real constant) [Groom and Bailey 1989], which is denoted by ' $S$ '. The static shift in the dual logarithmic scale will be $\log (S)$, as shown in Figure 1. This galvanic distortion can be modeled precisely in two dimensions and three dimensions by considering the local inhomogeneities causing the static shift [Bahr 1991]. However, also using a one-dimensional (1-D) formulation, the static shift can be modeled as a constant real number that is basically the shift in the apparent resistivity data.

Now the static shift ' $S$ ' is also considered as one of the model parameters and is optimized along with the other model parameters using global optimization. Static distortions of the magnetotelluric impedance tensor due to shallow 3-D electrical inhomogeneities were formally described by Cerv et al. [2010], as:

$$
Z_{\text {obs }}(r, T)=A_{\text {dist }}(\mathrm{r}) Z_{\text {reg }}(r, T),
$$

where $A_{\text {dist }}(r)$ is a frequency-independent distortion tensor, and $Z_{o b s}(r, T)$ and $Z_{\text {reg }}(r, T)$ are the observed and regional impedance tensors, respectively, at location $r$ and for period $T$. Although the actual expression for the static-shift-affected data is complex [Bahr 1991] for 2-D and 3-D structures, a formal expression in 1-D for the static-shift-affected apparent 
resistivity can be written in the following form (multiplication by a frequency-independent real constant) [Groom and Bailey 1989]:

$$
\rho_{a}^{\star}=\frac{S}{\mu_{0} \omega}\left|\frac{E}{H}\right|^{2}=\frac{S}{\mu_{0} \omega}\left|Z_{0}\right|^{2}
$$

Taking the log of both sides yields:

$$
\begin{gathered}
\log \left(\rho_{a}^{*}\right)=\log (S)+\log \left\{\frac{1}{\mu_{0} \omega}\left|\frac{E}{H}\right|^{2}\right\} \\
\log \left(\rho_{a}^{*}\right)=\log (S)+\log \left(\rho_{a}^{\text {true }}\right)
\end{gathered}
$$

However, the phase is not affected by the static shift and can be written as:

$$
\phi=\arg \left(Z_{0}\right)=\tan ^{-1}\left[\frac{\operatorname{Im}\left(Z_{0}\right)}{\operatorname{Re}\left(Z_{0}\right)}\right]
$$

The apparent resistivity and phase over the 1-D multilayered Earth is computed using the recursive relation for impedance, $Z_{0}$, given by Vozoff and Jupp [1975]:

$$
\begin{gathered}
Z_{1}=\frac{Z_{i+1}+T_{i}}{Z_{i+1} \times S_{i}+1} \quad \text { where } i=n-1, n-2, \ldots 0 \\
T_{i}=k \sqrt{\rho_{i}} \tanh \left[\frac{k h_{i}}{\rho_{i}}\right] \\
S_{i}=\frac{1}{k \sqrt{\rho_{i}}} \tanh \left[\frac{k h_{i}}{\rho_{i}}\right] \\
Z_{n}=-\frac{i \mu_{0} \omega}{v_{n}}==k \sqrt{\rho_{n}}
\end{gathered}
$$

where $k=\sqrt{-i \mu_{0} \omega}, \omega=2 \pi f, f$ is the frequency, and $\rho_{i}$ and $h_{i}$ are the resistivity and thickness of the various layers.

\section{The very fast simulated}

\section{annealing nonlinear optimization method}

\subsection{Basic concept}

Global optimization methods such as simulated annealing, genetic algorithms and artificial neural networks have been applied in multiparametric optimization of various geophysical datasets [e.g., Rothman 1985, Rothman 1986, Dosso and Oldenburg 1991, Sen et al. 1993, Sen and Stoffa 1995, Sharma and Kaikkonen 1998, Sharma and Kaikkonen 1999, Vedanti et al. 2005, Juan et al. 2010]. In the present study, VFSA optimization is used as it is more efficient and it takes less computing time in comparison to other global optimization algorithms [Sen and Stoffa 1995, p. 267]. Global optimization techniques require minimum and maximum limits (search range) for each model parameter. These limits are selected carefully. Measured apparent resistivity data is analyzed to decide on the number

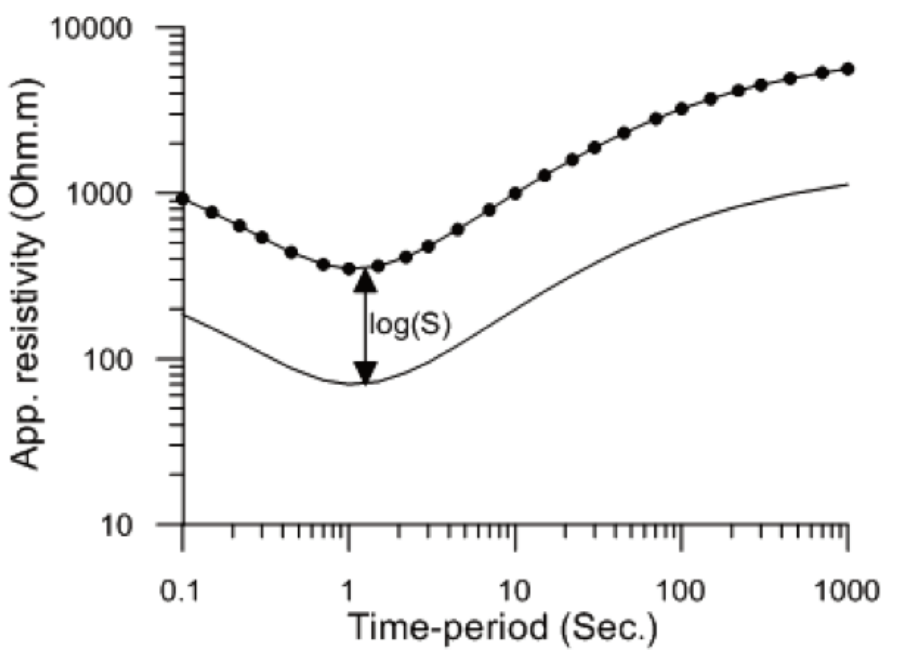

Figure 1. Example of static-shift-affected apparent resistivity data.

of layers and approximate resistivity of each layer. As resistivity varies over a large range, initially a large search range for resistivity is selected. The thickness variation is estimated from the inflection points on apparent resistivity sounding curves. Limits for the static shift can be set by a-priori information about the top-layer resistivity (either geological information or direct current resistivity measurements) in the study area, and comparing it with the field magnetotelluric apparent resistivity data. Correct estimations of the search range become more crucial when modeling static-shift-affected magnetotelluric data, because the actual resistivity and the measured apparent resistivity can be very far apart for static-shift-affected data. Therefore, the initial resistivity and the thickness search range is kept quite large. After a test run, the search limits can be modified to obtain better solutions. The optimization process tries to find the best-fit models in the selected model space. The objective function and governing equations of the VFSA processes are presented systematically below.

Initially, a model $P_{j}$ is selected randomly in the model space $P_{j}^{\text {min }} \leq P_{j} \leq P_{j}^{\max }(j=1 \ldots M)$, where $M$ is the total number of model parameters, an $L$-layer model consists of $L$ resistivity parameters $\left(\rho_{l}, l=1 \ldots L\right)$ and $L-1$ thickness parameters $\left(h_{l}, l=1 \ldots L-1\right)$, and the last model parameter $P_{M}$ represents the static shift and is a real constant ' $S$ ', thus the total number of model parameters $M=L+(L-1)+1=2 L$ ). The following objective function (misfit-error) $\varepsilon$ is calculated:

$$
\begin{gathered}
\varepsilon=\frac{1}{n} \sum_{i=1}^{n}\left[\ln \left(\rho_{i}^{0}\right)-\left\{\ln \rho_{i}^{c}\left(P_{j}, j=1, M-1\right)+\ln (S)\right\}\right]^{2}+ \\
+\frac{1}{n} \sum_{i=1}^{n}\left[\frac{\phi_{i}^{0}-\phi_{i}^{c}\left(P_{j}, j=1, M-1\right)}{\phi_{i}^{0}}\right]^{2}
\end{gathered}
$$

where, $\rho_{i}^{0}$ and $\rho_{i}^{c}\left(P_{j}\right)$ are the $i^{\text {th }}$ observed and model responses, respectively, which represent the apparent resistivity. $\phi_{i}^{0}$ and $\phi_{i}^{c}\left(P_{j}\right)$ are the $i^{\text {th }}$ observed and model responses, respectively, which represent the phase. $N$ is the number of observation 
points. While doing individual inversions, the objective function dealing with corresponding terms in Equation (5) are considered.

The parameters and the objective function of the abovecomputed model are kept in memory and each parameter is perturbed according to the Cauchy probability distribution. The updating factor $y_{j}$ for the $j^{\text {th }}$ parameter is computed from the equation:

$$
y_{j}=\operatorname{sgn}\left(u_{j}-0.5\right) T_{j}\left[\left[1+\frac{1}{T_{j}}\right]^{\left|2 u_{j}-1\right|}-1\right]
$$

such that $y_{j}$ varies between -1 and +1 . In the above equation, $\mathcal{u}_{j}$ is a random number and varies between 0 and $1, T_{j}$ is the temperature, which can be the same or different for various parameters, depending on the nature of the problem. Each parameter $P_{j}$ is updated by the equation:

$$
P_{j}^{k k+1}=P_{j}^{k k}+y_{j}\left(P_{j}^{\max }-P_{j}^{\min }\right)
$$

where $P_{j}^{k k+1}$ is the new model parameter computed with respect to its previous value $P_{j}^{k k}$ ( $k k$ represents successive movement in the model space). Hence, a new model is obtained. Now the objective function for the new model is calculated and compared with the previous model. If the misfit-error of the new model is less than the misfit-error for the previous model, then the new model is selected with the probability $\exp (-\Delta \varepsilon / T)$, where $\Delta \varepsilon$ is the difference for the objective functions of both models. When the misfit-error of the new model is higher than that of the previous model, then a random number is drawn and compared with the probability. If the probability is greater than the random number drawn, then also the new model is accepted with the same probability, otherwise this new model is rejected, keeping the previous model and its objective function in memory. Next, the desired number of moves is made at the same temperature level by accepting and rejecting the new models according to above-mentioned criterion, and this completes a single iteration. Movement in the model space at one temperature level produces an improved model. After completing the desired number of moves at the particular temperature, the temperature is reduced to a lower level according to the following cooling schedule:

$$
T_{j}(k)=T_{0 j} \exp \left(-c_{j} k^{1 / M}\right)
$$

where $k$ is the number of iterations, $c_{j}$ is a problemdependant constant that can vary for different model parameters. $T_{0 j}$ is the initial temperature that can also be different for various parameters and its initial value depends on the nature of the objective function considered for the optimization. $M$ is the number of model parameters. In the present study, $c_{j}=1$ and the initial temperature $T_{0 j}=0.1$ is selected. The final temperature in the simulated annealing should be lowered by $4-5$ orders of magnitude with respect to the initial temperature. If we use $1 / M$ according to the number of model parameters, then the process takes a very long time to reach the final temperature. To avoid this, $1 / M$ is replaced by a suitable fraction, so that within $500-1000$ iterations the desired lower temperature is reached [Sharma and Kaikkonen 1998]. The factor 0.4 is suitable for the individual and joint optimization of the apparent resistivity and phase data.

After lowering the temperature, the desired number of moves with the selection criterion described above is repeated at the lower temperature level. Subsequently, the temperature is reduced gradually using Equation (8), to a sufficiently low value, to obtain better and better models at each temperature level. After completion of the desired iterations (say 500 or 1000), a single solution is obtained.

Once a solution is obtained, we compare the estimated model parameters with the minimum and maximum limits for each model parameter. If any model parameter is located on the boundary of the search range, then the search range of that parameter is modified accordingly. If the resistivity of a layer is optimized as 50 $\Omega \mathrm{m}$ and lower limit of that layer is also set at $50 \Omega \mathrm{m}$, for example, this means that the actual value of resistivity might be lower than $50 \Omega \mathrm{m}$ and hence the lower limit should be decreased. In the same way for all of the parameters, the optimized model parameter is checked to see that it lies within the minimum and maximum limits defined for each mode parameter. After a satisfactory solution is obtained, the VFSA procedure is repeated a number of times. The search range can also be restricted after this test run.

The whole procedure is repeated a number of times to obtain more solutions, and every time the process is initialized at different randomly selected locations in the predefined model space. It is important to mention that at the initially higher temperatures, the accepted model parameters vary over a large range. This is because in a highly complex multidimensional error (misfit) surface the probability density function is also as complex as the misfit surface. However, as the temperature decreases, the probability density function becomes smoother and shows sharper peaks. Hence, the accepted model parameters become localized near the well-defined peaks and have a tendency to cluster around the minima. At sufficiently low temperatures, the algorithm accepts models in the vicinity of the global minima, where the probability density function has a sharp peak and other local minima are absolutely invisible in the probability density function.

It should be emphasized here that static shifts evaluated in various solutions can be different, although they will cluster around a point. The mean of all of these values will be very close to the actual static shift in the data. 


\begin{tabular}{|c|c|c|c|c|c|}
\hline Parameters & True values & Search range & App. Resistivity & Phase & Joint \\
\hline$\rho_{1}(\Omega \mathbf{m})$ & 500 & $100-5000$ & $3651.4 \pm 1955$ & $1756.3 \pm 727$ & $2575.8 \pm 960$ \\
\hline $\boldsymbol{\rho}_{2}(\boldsymbol{\Omega m})$ & 50 & $10-500$ & $248.5 \pm 8.7$ & $175.9 \pm 74.5$ & $250.4 \pm 15.5$ \\
\hline $\boldsymbol{\rho}_{3}(\mathbf{\Omega m})$ & 1500 & $1000-10000$ & $7498.4 \pm 19.6$ & $5282.7 \pm 2250$ & $7496.4 \pm 590$ \\
\hline $\mathbf{h}_{1}(\mathbf{m})$ & 1000 & $500-5000$ & $2252.35 \pm 250$ & $1835.51 \pm 409$ & $2227.7 \pm 150$ \\
\hline $\mathbf{h}_{2}(\mathbf{m})$ & 3000 & $1000-10000$ & $6665.43 \pm 392$ & $5499.3 \pm 1172$ & $6725.2 \pm 600$ \\
\hline$S$ & 5 & - & - & - & - \\
\hline Misfit error & - & - & $1.14 \times 10^{-5}$ & $9.31 \times 10^{-5}$ & $2.30 \times 10^{-8}$ \\
\hline
\end{tabular}

Table 1. Model 1: inversion results without optimization of static shift.

\subsection{Mean model, covariance and correlations}

A single run of global converging algorithms is not sufficient to find the global solution [e.g., Sen and Stoffa 1995]. However, the model parameters obtained through several runs can differ from each other. Therefore, a statistical mean model is computed. Computation of the mean model is based on the assumption that the models obtained by various VFSA runs will lie in close proximity and will have a Gaussian distribution. The mean model $\bar{P}_{j}$ is computed from various solutions to obtain the global model, using the formulation [e. g., Tarantola 1987]:

$$
\bar{P}_{j}=\frac{1}{N R} \sum_{k=1}^{N R} P_{j, k} \exp \left(-\varepsilon_{k}\right)
$$

where NR is the number of runs performed, and $\varepsilon_{k}$ is the misfiterror obtained at the $k^{\text {th }}$ run. Subsequently, the covariance and correlation matrices are also computed by the equations:

$$
\operatorname{Cov} P(i, j)=\frac{1}{N R} \sum_{k=1}^{N R}\left(P_{i, k}-\bar{P}_{i}\right)\left(P_{j, k}-\bar{P}_{j}\right) \exp \left(-\varepsilon_{k}\right)
$$

and

$$
\operatorname{Cov} P(i, j)=\frac{\operatorname{Cov} P(i, j)}{\sqrt{\operatorname{Cov} P(i, i) \times \operatorname{Cov} P(j, j)}} .
$$

In Equations (10) and (11), $i$ and $j$ vary from 1 to $M$. Ten VFSA runs are performed for a single model, and thus the model parameters obtained are used to compute the mean model, and the covariance and correlation matrices. The square roots of the diagonal elements of the covariance matrix represent the uncertainties in the mean-model parameters; hence, the covariance matrix can be used to find the uncertainties in the mean model. The correlation matrix computed from the covariance matrix expresses the relationship between the parameters and the associated physics.

\section{Results}

Initially, synthetic and noisy datasets with different magnitudes of static shift are generated using Equation (1) for a number of models. These datasets are optimized using
VFSA global optimization, to retrieve the model parameters and the static shift that was introduced to generate the data. For any particular model, three-types of inversions are performed. First, the apparent resistivity data are used in the inversion. Next, the phase data are inverted, and finally, joint inversions of the apparent resistivity and phase data are performed. Initially, the static-shift-affected data are inverted without optimizing the static shift. Subsequently, the same data are inverted considering the static shift as a model parameter in the global optimization. The resistivity and thickness of the various layers obtained from the inversions are compared with the actual model parameters, and an analysis is presented to obtain accurate estimations of the model parameters from the static-shift-affected magnetotelluric data. In the absence of static shift, the value of ' $S$ ' will be unity. If ' $S$ ' is substituted by 1 in Equation (1), then it changes to the conventional equation for the apparent resistivity in the magnetotelluric method. Even if there is no static shift in the data, the proposed approach can still be used to optimize the static shift. The estimated value of ' $S$ ' under such conditions will be close to unity.

In the inversions demonstrated below, the static-shiftaffected data are used for all of the models, except model 4, in which a different situation has been modeled.

\subsection{Model 1}

The first model shows a three-layer structure with a sandwiched conductive layer. The static shift factor $S=5$ is used to generate the static-shift-affected data. Table 1 shows the true model, the search range for each model parameter, and the estimated model parameter (for each inversion), without optimizing the static shift. Through analysis of the model parameters shown in Table 1 (and especially $\rho_{1}$ ) and the apparent resistivity data shown in Figure 2a, the role of the static shift parameter in affecting the data generated can be easily understood.

Figure 2a shows the fit between the observed and model data after the individual inversion of the apparent resistivity data without optimization of the static-shift 

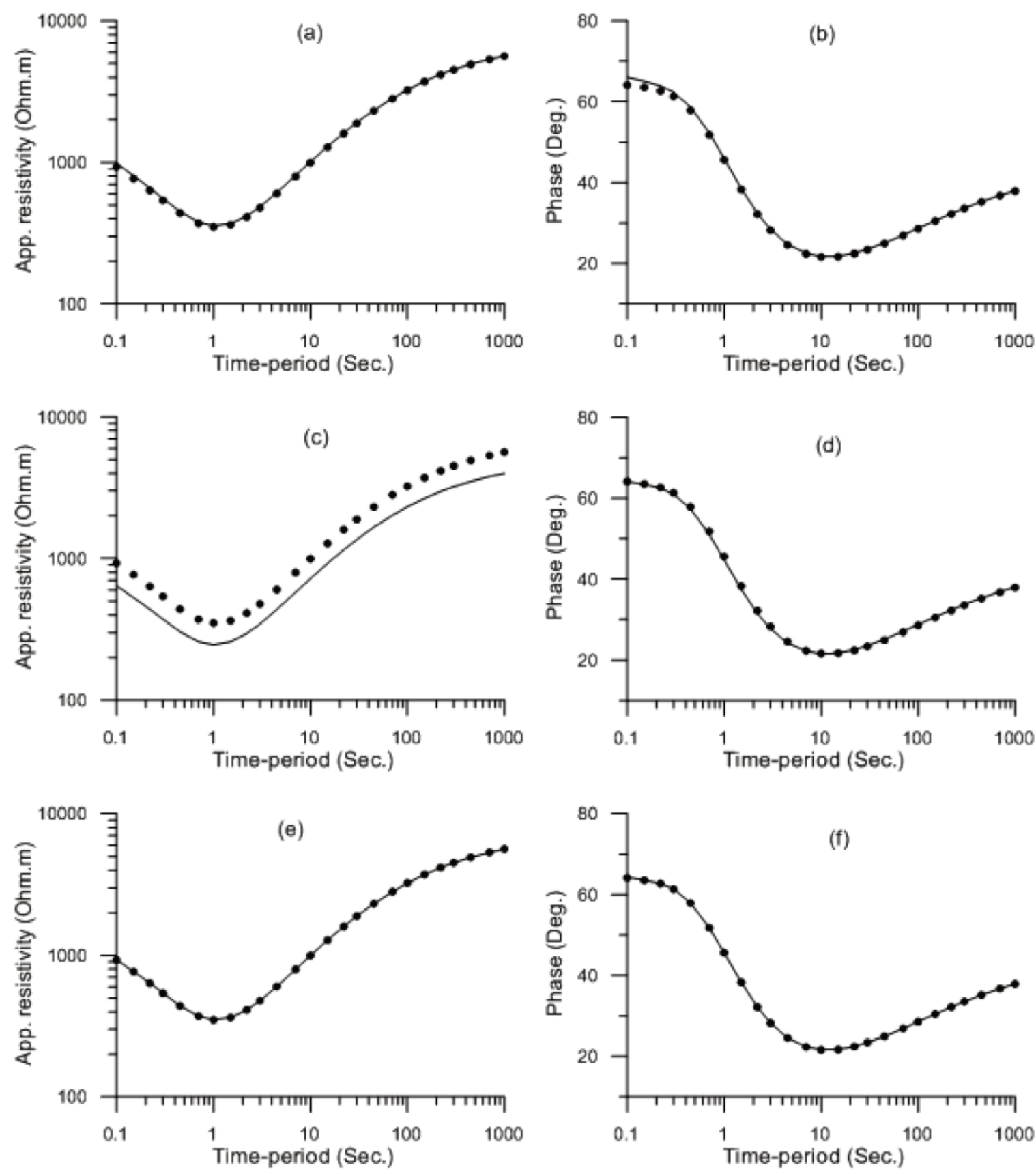

Figure 2. Comparisons between observed data (solid symbols) and model data (lines) for model 1 after inversion of the apparent resistivity data (a, b), the phase data (c, d) and both sets of data $(e, f)$.

parameter. The mean model obtained after this individual inversion of the apparent resistivity data is also used to compute the corresponding phase data, and it is compared to the observed phase data in Figure $2 \mathrm{~b}$. It is evident from Figure 2a, $b$ that even though the observed data are affected by static shift, the mean model generated without optimization of the static shift fits very well with the observed data. Although the phase data is not used in this inversion, the mean model obtained after the inversion of the apparent resistivity data also fits the observed phase data very well. The model parameters estimated from individual inversions of the apparent resistivity data yield erroneous results, as expected (Table 1).
Figure $2 \mathrm{~d}$ shows the corresponding fits between the observed and the model data after the individual inversion of the phase data. The models obtained after inversion of the phase data are used to compute the apparent resistivity data. The computed apparent resistivity data are compared with the observed apparent resistivity data. It is evident from Figure $2 \mathrm{c}$ that the model responses do not fit with the observed apparent resistivity data. A parallel shift is clearly evident in Figure 2c. Comparison of the inverted model parameters obtained after the individual inversion of the phase and apparent resistivity data shown in Table 1 suggests that individual inversions of phase data yield better estimations of the model parameters than the individual 


\begin{tabular}{cccccc}
\hline Parameters & True values & Search range & App. Resistivity & Phase & Joint \\
\hline $\boldsymbol{\rho}_{\mathbf{1}}(\mathbf{\Omega})$ & 500 & $100-5000$ & $206.03 \pm 188$ & $406.93 \pm 45$ & $428.89 \pm 61$ \\
$\boldsymbol{\rho}_{\mathbf{2}}(\mathbf{\Omega})$ & 50 & $10-500$ & $40.04 \pm 9.4$ & $41.2 \pm 5.5$ & $49.28 \pm 9.9$ \\
$\mathbf{\rho}_{\mathbf{3}}(\mathbf{\Omega})$ & 1500 & $1000-10000$ & $1474.16 \pm 202$ & $1241 \pm 177$ & $1571.98 \pm 44$ \\
$\mathbf{h}_{\mathbf{1}}(\mathbf{m})$ & 1000 & $500-5000$ & $1398.21 \pm 333$ & $913.63 \pm 76$ & $1121.53 \pm 261$ \\
$\mathbf{h}_{\mathbf{2}}(\mathbf{m})$ & 3000 & $1000-10000$ & $2235.21 \pm 591$ & $2709 \pm 161$ & $2830.69 \pm 196$ \\
$\mathbf{S}$ & 5 & $1-10$ & $5.15 \pm 0.70$ & $3.94 \pm 1.26$ & $4.74 \pm 0.63$ \\
$\mathbf{M i s f i t}$ error & - & - & $1.81 \times 10^{-5}$ & $8.45 \times 10^{-7}$ & $1.09 \times 10^{-6}$ \\
\hline
\end{tabular}

Table 2. Model 1: inversion results with optimization of static shift.

inversions of the apparent resistivity data. However, due to nonuniqueness, the model parameters obtained after individual inversions of the phase data are far from the actual model. Hence, even though the individual inversion of phase data can predict the presence of static shift, it cannot yield an accurate estimation of the model parameters. If we remove the static shift that is visible in Figure $2 \mathrm{c}$ by matching the measured apparent resistivity data (dots) with the mean model responses obtained by individual inversion of the phase data (solid line), and perform individual or joint inversion, then we will also not be able to get the actual model parameters. This is because the apparent resistivity data generated from the mean model obtained by the individual inversion of the phase data still remains highly inaccurate. The revised apparent resistivity data can certainly improve the results compared to the individual inversion of the apparent resistivity data affected by static shift, but it will still yield inaccurate subsurface structure.

Fits between the observed and model data after the joint inversion of the apparent resistivity and phase data are presented in Figure 2e, f. The observed and model data fit extremely well after the joint inversion. Analysis of the results presented in Table 1 shows that if the joint inversion of the synthetic data is performed without taking the static shift into consideration, then the estimated resistivities are multiplied by a factor that is approximately equal to $S(S=5$ in this case) and the estimated thicknesses are multiplied by a factor that is almost equal to $\sqrt{ } S(\sqrt{5}=2.23)$. Although the static shift is not optimized, the data for the estimated model fits the observed data very well. This is also evident from the very small misfit errors shown in Table 1 . Moreover, Table 1 reveals that incorrect estimations of the model parameters are obtained by optimizing the static-shift-affected data. The relation between the static shift introduced in the synthetic data and the estimated model parameters is in agreement with previous studies [Tournerie et al. 2007, among others]. This reveals that the present approach yields the expected result and that it can be performed to optimize the static shift along with the other model parameters, to obtain reliable estimations of the subsurface structure.

Subsequently, the static shift is considered as one of the model parameters for optimization. Individual and joint inversions of the apparent resistivity and phase data are performed. Plots similar to Figure 2 were generated in this case too, and both of these plots looked identical. For example, the mean model obtained by individual inversion of the apparent resistivity data fits exactly the apparent resistivity data as well as the phase data. Individual inversion of the phase data fits only the measured phase data and shows a parallel shift for the apparent resistivity data (as in Figure 2c), and the joint inversion fits both sets of data. Therefore, for brevity, the corresponding plot is not shown, and only the numerical results are given in Table 2. Individual inversions of the apparent resistivity data yield better estimations when the static shift is also optimized as a model parameter (Table 2), compared to when it is not considered as a model parameter (Table 1). Moreover, this still yields inaccurate estimations of the subsurface structure. Individual inversion of the phase data with the static shift optimization also yields better results and misfit errors. It is important to mention here that while carrying out individual inversions of the phase data, optimization of the static shift does not have any significance. Therefore, the final model after individual inversion of the phase data in Tables 1 and 2 should be the same. However, these values are different. The reason for this difference is that the static shift is involved while matching the apparent resistivity data when the phase data is inverted, although it has no role in the misfit error calculation. It is evident from Tables 1 and 2 that the misfit error is very small after individual inversion of the phase data, although the models are widely different. This shows the degree of nonuniqueness of the individual inversion results using phase data. Joint inversion of the apparent resistivity and phase data using static shift as a model parameter for optimization yields the best results. All of the model parameters, along with the static shift, are estimated 
as very close to their true values (Table 2). The uncertainties shown in Table 2 for most of the model parameters are smaller for joint inversion as compared to the individual inversion results.

\subsection{Model 2}

The second model depicts a four-layer Earth structure with a conductive and a resistive sandwiched layer. We used a different static shift parameter $(S=3)$ for this model, to generate synthetic responses compared to model 1 . However, the search range of the static shift is kept within 1-10, as in the previous model. This is to examine whether the estimation of the static shift gets closer to the actual static shift introduced in the data, or whether it is just a random number generated in the range $1-10$. The true values, as the search range and estimated model parameters without optimizing static shift, are given in Table 3 .

Individual inversions of the apparent resistivity data (without considering static shift) once again yield the mean model, which fits very well with the measured apparent resistivity as well as with the phase data (Figure 3a, b). The mean model parameters are widely different compared to the actual values (Table 3). The responses (as apparent resistivity and phase) for the mean model obtained after individual inversion of the phase data only fits the observed phase data, and does not fit the apparent resistivity data (Figure 3c, d). After inversion of the phase data, a parallel shift (Figure 3c) reveals that the static shift is present in the apparent resistivity data. Even though Figure $3 \mathrm{~d}$ shows a good fit between the model and the observed phase data, the misfit error in Table 3 is relatively large. The reason for this could be that the different solutions derived from the inversion of the individual phase data might not lie in the same region. If some of the models are located significantly away from the other models, then the computed mean model might deviate in such a way that the misfit error could change and the mean model response might not fit the observation at all. However, the presented misfit error is still very small, and the mean model response fits very well with observation. Joint inversion of the apparent resistivity and phase data without taking the static shift into consideration yields the estimated resistivities that are multiplied by an amount approximately equal to the value of $S(=3)$ and the estimated thicknesses that are multiplied by an amount approximately equal to $\sqrt{3}(=1.73)$. The mean model responses fit very well with the observed apparent resistivity and phase data (Figure 3e, f).

Next, individual and joint inversions of the static-shiftaffected data are performed by optimizing the static shift along with the other model parameters. Since we are not presenting the corresponding figures for brevity, the numerical value of the misfit error presented in Table 4 can be compared, to assess the fits between the observed and model responses. We can see that the misfit errors are comparable for individual and joint inversion, and they are also significantly small enough to predict that the fits between the observed and model data are very good. The interpreted mean model parameters shown in Table 4 reveal that due to the insensitivity of the electromagnetic methods towards a resistive layer, uncertainties in the resistivity of the resistive layers are larger in comparison to those of the conducting layer. The results of the comparisons of individual and joint inversion in Table 4 reveal that joint inversion predicts the static shift and the other model parameters very close to the actual values. The uncertainties presented in Tables 3 and 4 without or with static-shift optimization reveal that this is smaller for most of the parameters after the joint inversion, as compared to the individual inversions.

It is worth highlighting that a large search range for the various model parameters is considered, because the data are affected by the static shift. Various solutions obtained by

\begin{tabular}{cccccc}
\hline Parameters & True values & Search range & App. Resistivity & Phase & Joint \\
\hline $\boldsymbol{\rho}_{\mathbf{1}}(\mathbf{\Omega})$ & 200 & $100-1000$ & $565.3 \pm 169$ & $370.8 \pm 137$ & $572.46 \pm 152$ \\
$\boldsymbol{\rho}_{\mathbf{2}}(\mathbf{\Omega} \mathbf{m})$ & 1000 & $100-10000$ & $7306 \pm 2796$ & $4352 \pm 2774$ & $4410.3 \pm 2164$ \\
$\boldsymbol{\rho}_{3}(\boldsymbol{\Omega} \mathbf{m})$ & 20 & $1-100$ & $77.23 \pm 24.2$ & $40.81 \pm 18$ & $74.62 \pm 14$ \\
$\boldsymbol{\rho}_{4}(\mathbf{\Omega})$ & 5000 & $1000-20000$ & $14950.4 \pm 64$ & $9518 \pm 2080$ & $14971 \pm 1265$ \\
$\mathbf{h}_{\mathbf{1}}(\mathbf{m})$ & 1000 & $500-3000$ & $1908.6 \pm 687$ & $1570.6 \pm 626$ & $1813.7 \pm 655$ \\
$\mathbf{h}_{\mathbf{2}}(\mathbf{m})$ & 5000 & $1000-10000$ & $8031.7 \pm 876$ & $6526.08 \pm 1007$ & $8241.6 \pm 929$ \\
$\mathbf{h}_{\mathbf{3}}(\mathbf{m})$ & 2000 & $1000-5000$ & $4564.4 \pm 1486$ & $3017.86 \pm 1224$ & $4362.7 \pm 1167$ \\
$\mathbf{S}$ & 3 & $1-10$ & - & - & - \\
Misfit error & - & - & $2.57 \times 10^{-6}$ & $1.26 \times 10^{-4}$ & $2.96 \times 10^{-6}$ \\
\hline
\end{tabular}

Table 3. Model 2: inversion results without optimization of static shift. 

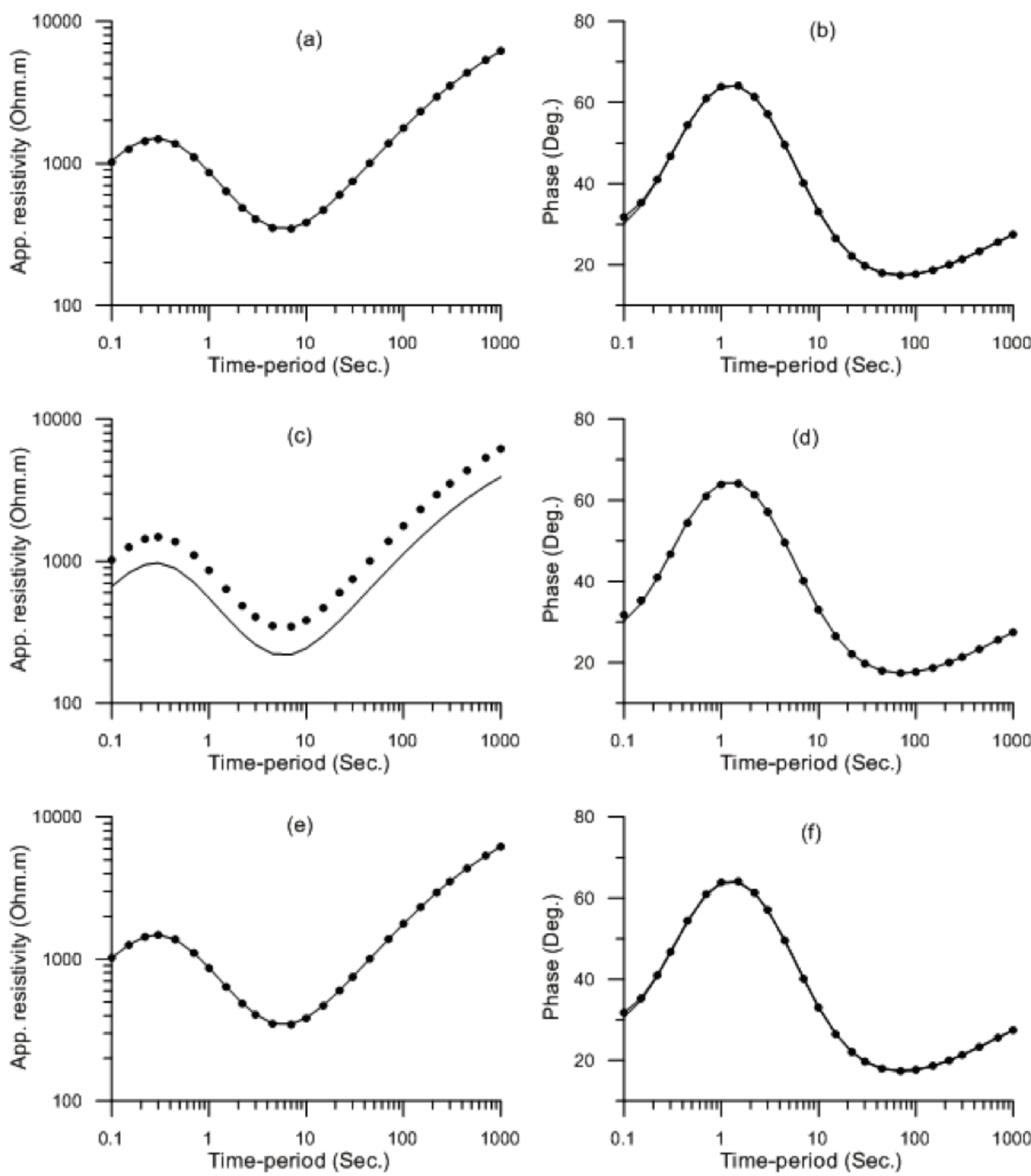

Figure 3. Comparisons between observed data (solid symbols) and model data (lines) for model 2 after inversion of the apparent resistivity data (a, b), the phase data (c, d) and both sets of data (e, f).

\begin{tabular}{cccccc}
\hline Parameters & True values & Search range & App. Resistivity & Phase & Joint \\
\hline $\boldsymbol{\rho}_{\mathbf{1}}(\mathbf{\Omega})$ & 200 & $100-1000$ & $186.6 \pm 53$ & $131.7 \pm 39$ & $162.67 \pm 39$ \\
$\boldsymbol{\rho}_{\mathbf{2}}(\mathbf{\Omega})$ & 1000 & $100-10000$ & $1704.7 \pm 721$ & $564.2 \pm 949$ & $1354.7 \pm 934$ \\
$\boldsymbol{\rho}_{\mathbf{3}}(\mathbf{\Omega})$ & 20 & $1-100$ & $23.36 \pm 7.5$ & $11.44 \pm 6.2$ & $22.06 \pm 7.6$ \\
$\boldsymbol{\rho}_{\mathbf{4}}(\mathbf{\Omega})$ & 5000 & $1000-20000$ & $4663.2 \pm 765$ & $3748 \pm 767$ & $4304 \pm 1083$ \\
$\mathbf{h}_{\mathbf{1}}(\mathbf{m})$ & 1000 & $500-3000$ & $1101.4 \pm 350$ & $604.3 \pm 398$ & $909.70 \pm 251$ \\
$\mathbf{h}_{\mathbf{2}}(\mathbf{m})$ & 5000 & $1000-10000$ & $4343.5 \pm 594$ & $4776 \pm 796$ & $4355.9 \pm 801$ \\
$\mathbf{h}_{\mathbf{3}}(\mathbf{m})$ & 2000 & $1000-5000$ & $2474.4 \pm 822$ & $1301 \pm 634$ & $2410 \pm 684$ \\
$\mathbf{S}$ & 3 & $1-10$ & $3.25 \pm 0.53$ & $3.78 \pm 1.2$ & $3.4 \pm 0.7$ \\
$\mathbf{M i s f i t}$ error & - & - & $4.44 \times 10^{-6}$ & $1.29 \times 10^{-6}$ & $1.06 \times 10^{-5}$ \\
\hline
\end{tabular}

Table 4. Model 2: inversion results with optimization of static shift. 
global inversion are widely spread in this large model space. Therefore, the mean model derived from these solutions reveals large uncertainties in each of the model parameters. As joint inversion of the apparent resistivity and phase data yields the most accurate results, while also optimizing the static shift, this process can be repeated using a smaller model space that is based on uncertainties in each model parameter. This yields the best results, with very small uncertainties in each model parameter [Sharma and Verma 2003].

\subsection{Model 3}

The third model also depicts a 4-layer Earth model with a sandwiched resistive and conductive layer. Random noise is superimposed on the synthetic data to simulate field data. This is intended to demonstrate the applicability of the approach developed for optimizing the static shift along with the resistivity and thickness for the field data. We applied $10 \%$ random noise on the synthetic data, such that the mean and standard deviation of the error introduced was zero. The true model parameters, the search range, and the estimated model parameters are given in Table 5.

Individual and joint inversions without and with optimizing the static shift are performed with the noisy synthetic data. Figure 4 shows the similar fits between the observed and the model data, which has been seen for previous models. When the static shift is not considered in the optimization, the estimated model parameters are far from their true values, even though the mean model data fits very well with the observed data. After the joint inversion, the estimated resistivities are multiplied by an amount that is approximately equal to the value of $S(=6)$ and the estimated thicknesses are multiplied by an amount approximately equal to $\sqrt{6}(=2.45)$. When the static shift is optimized as a model parameter, then the estimated model parameters are very close to their actual values after the joint inversion (Table 6). Even though we consider the static-shift optimization, the individual inversion results are not reliable. It can also be seen that in all the results of the inversion (Tables 1 to 6 ), the resistivity of a conductive sandwiched layer is resolved better than the resistive sandwiched layer. This is because the magnetotelluric method, or in general, electromagnetic methods, are sensitive towards conductive structures.

\subsection{Model 4}

The study presented for Model 4 depicts a special condition. It assumes that the measured apparent resistivity data is not affected by the static shift, and by using the present approach, the static shift is optimized for this data. Under such circumstances, it is worth investigating how the inverted model parameters will be affected. To model this, the true value of ' $S$ ' in this model is taken as 1 (or free of static shift). As the nature of the noise in the data is never known, for this model, $10 \%$ Gaussian noise (with mean and standard deviation zero) is added to the data to simulate field conditions.

Inversion results for this model are presented in Figure 5 and Table 7. Individual inversions of the apparent resistivity data yield lower estimates of resistivities. Accordingly, the estimated thickness is also lower than the actual value (especially for the second layer). However, this is in agreement with the principle of equivalence in electromagnetic sounding curves. Furthermore, the individual inversion of the phase data yields higher estimates of the resistivities. Therefore, neither the apparent resistivity nor phase data yield the actual model when the static shift is optimized as a model parameter for a dataset that is actually not affected by static shift. Interestingly, after joint inversion (Table 7), the resistivities and thicknesses of all of the layers are well estimated, as close to their respective true values. The apparent resistivity and phase data fit very well (Figure 5e, f) with the respective measured data. Here again, it has been observed that when the phase data is inverted, the apparent

\begin{tabular}{cccccc}
\hline Parameters & True values & Search range & App. Resistivity & Phase & Joint \\
\hline $\boldsymbol{\rho}_{\mathbf{1}}(\mathbf{\Omega})$ & 5000 & $1000-30000$ & $23109 \pm 6937$ & $16556 \pm 5623$ & $27484 \pm 2568$ \\
$\boldsymbol{\rho}_{\mathbf{2}}(\mathbf{\Omega})$ & 20 & $1-200$ & $106.38 \pm 28$ & $65.54 \pm 21.94$ & $109.25 \pm 15$ \\
$\boldsymbol{\rho}_{3}(\mathbf{\Omega})$ & 2000 & $1000-20000$ & $13802 \pm 6740$ & $13350 \pm 6707$ & $12091 \pm 5861$ \\
$\boldsymbol{\rho}_{\mathbf{4}}(\mathbf{\Omega})$ & 10 & $1-200$ & $60.16 \pm 0.82$ & $33.35 \pm 12.33$ & $60.18 \pm 8.6$ \\
$\mathbf{h}_{\mathbf{1}}(\mathbf{m})$ & 4000 & $1000-20000$ & $10019 \pm 417$ & $7195 \pm 1391$ & $9922 \pm 176$ \\
$\mathbf{h}_{\mathbf{2}}(\mathbf{m})$ & 2000 & $1000-10000$ & $4308.2 \pm 1211$ & $3585.3 \pm 527$ & $4401.6 \pm 755$ \\
$\mathbf{h}_{\mathbf{3}}(\mathbf{m})$ & 20000 & $10000-50000$ & $49269 \pm 572$ & $35863 \pm 6972$ & $49333 \pm 557$ \\
$\mathbf{S}$ & 6 & $1-10$ & - & - & - \\
Misfit error & - & - & $6.32 \times 10^{-7}$ & $3.10 \times 10^{-5}$ & $4.25 \times 10^{-7}$ \\
\hline
\end{tabular}

Table 5. Model 3: inversion results without optimization of static shift. 

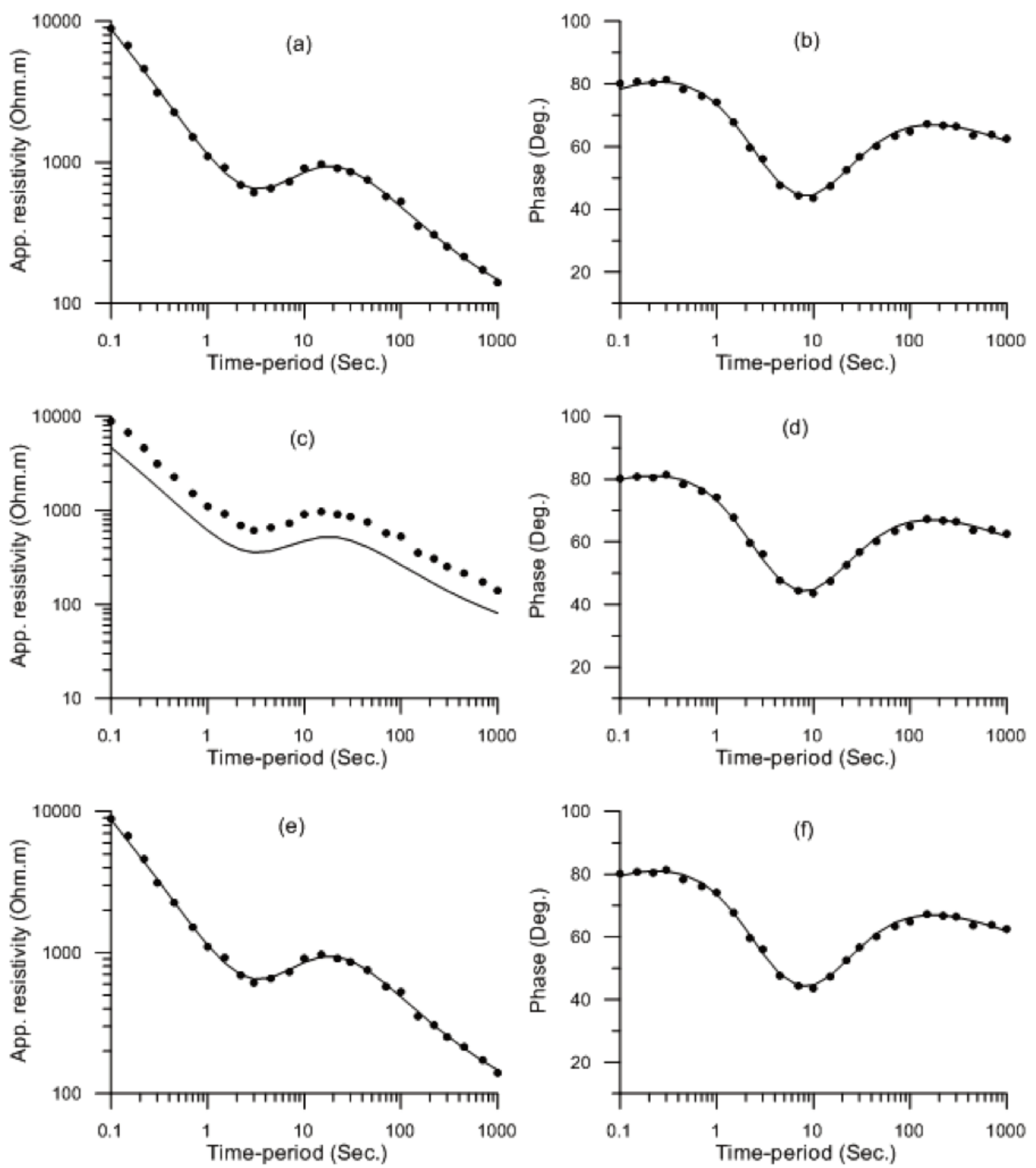

Figure 4. Comparisons between observed data (solid symbols) and model data (lines) for model 3 after inversion of the apparent resistivity data (a, b), the phase data (c, d) and both sets of data (e, f).

\begin{tabular}{cccccc}
\hline Parameters & True values & Search range & App. Resistivity & Phase & Joint \\
$\boldsymbol{\rho}_{\mathbf{1}}(\mathbf{\Omega})$ & 5000 & $1000-30000$ & $6812 . \pm 2374$ & $5444 \pm 516$ & $5330.8 \pm 708$ \\
$\boldsymbol{\rho}_{\mathbf{2}}(\mathbf{\Omega})$ & 20 & $1-200$ & $21.97 \pm 2.7$ & $21.49 \pm 2.7$ & $20.85 \pm 3.4$ \\
$\boldsymbol{\rho}_{\mathbf{3}}(\mathbf{\Omega})$ & 2000 & $1000-20000$ & $2005.9 \pm 612$ & $1977 \pm 768$ & $1779.05 \pm 686$ \\
$\boldsymbol{\rho}_{\mathbf{4}}(\mathbf{\Omega})$ & 10 & $1-200$ & $11.11 \pm 0.6$ & $11.14 \pm 0.6$ & $10.82 \pm 0.55$ \\
$\mathbf{h}_{\mathbf{1}}(\mathbf{m})$ & 4000 & $1000-20000$ & $4221.5 \pm 130$ & $4245.8 \pm 88$ & $4186.13 \pm 91$ \\
$\mathbf{h}_{\mathbf{2}}(\mathbf{m})$ & 2000 & $1000-10000$ & $2066.7 \pm 266$ & $2005 \pm 246$ & $1969.63 \pm 323$ \\
$\mathbf{h}_{\mathbf{3}}(\mathbf{m})$ & 20000 & $10000-50000$ & $21169.4 \pm 551$ & $21247 \pm 490$ & $20963.6 \pm 514$ \\
$\mathbf{S}$ & 6 & $1-10$ & $5.4 \pm 0.28$ & $4.98 \pm 1.64$ & $5.55 \pm 0.3$ \\
Misfit error & - & - & $7.06 \times 10^{-7}$ & $2.05 \times 10^{-6}$ & $4.08 \times 10^{-7}$ \\
\hline
\end{tabular}

Table 6. Model 3: inversion results with optimization of static shift. 

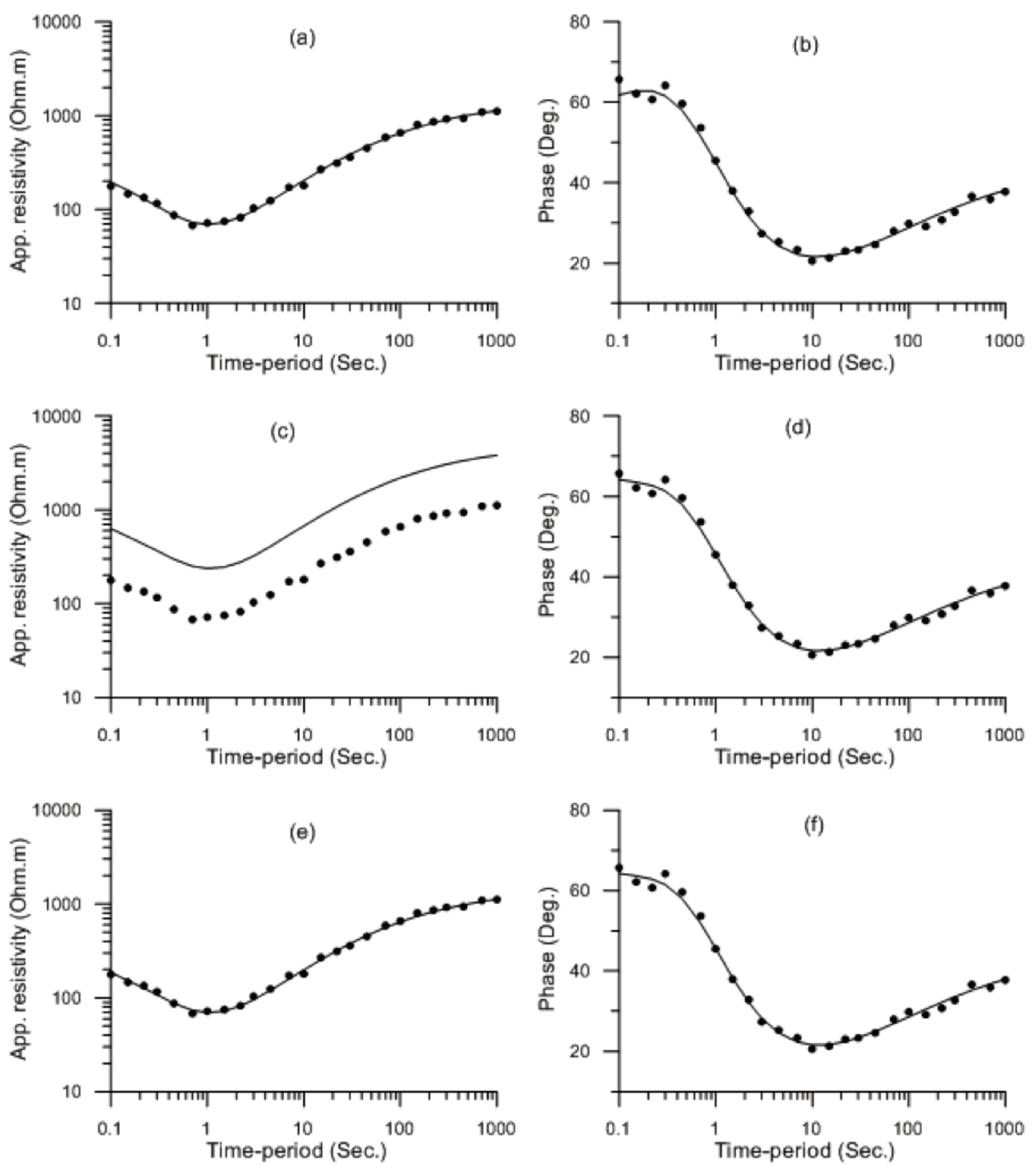

Figure 5. Comparisons between observed data (solid symbols) and model data (lines) for model 4 after inversion of the apparent resistivity data (a, b), the phase data $(c, d)$ and both sets of data $(e, f)$.

\begin{tabular}{cccccc}
\hline Parameters & True values & Search range & App. Resistivity & Phase & Joint \\
\hline $\boldsymbol{\rho}_{\mathbf{1}}(\mathbf{\Omega})$ & 500 & $100-5000$ & $250.4 \pm 150$ & $673 \pm 135$ & $489.48 \pm 65$ \\
$\boldsymbol{\rho}_{\mathbf{2}}(\mathbf{\Omega})$ & 50 & $10-500$ & $34.99 \pm 5.5$ & $67.3 \pm 13.7$ & $48.45 \pm 5.2$ \\
$\boldsymbol{\rho}_{\mathbf{3}}(\mathbf{\Omega})$ & 1500 & $1000-10000$ & $1241.08 \pm 128$ & $2018 \pm 413$ & $1463 \pm 150$ \\
$\mathbf{h}_{\mathbf{1}}(\mathbf{m})$ & 1000 & $500-5000$ & $1155.13 \pm 346$ & $1160 \pm 124$ & $994.22 \pm 55$ \\
$\mathbf{h}_{\mathbf{2}}(\mathbf{m})$ & 3000 & $1000-10000$ & $2153.8 \pm 486$ & $3480 \pm 362$ & $2940 \pm 186$ \\
$\mathbf{S}$ & 1 & $1-10$ & $1.2 \pm 0.13$ & $2.51 \pm 0.76$ & $1.02 \pm 0.1$ \\
$\mathbf{M i s f i t}$ error & - & - & $1.81 \times 10^{-5}$ & $2.71 \times 10^{-6}$ & $5.83 \times 10^{-8}$
\end{tabular}

Table 7. Model 4 (zero static shift): inversion results with optimization of static shift. 
resistivity curves do not fit very well (Figure 5c) with observation. This shows that even if the phase data is not affected by the static shift, the inversion of the phase alone will not lead to an accurate interpretation. The data in Table 7 show that even though they do not have any static shift, individual inversions of the apparent resistivity and phase data still predict that the data are affected by static shift. This shows the unreliability of the individual inversions. However, the joint inversion can clearly predict that the data is not affected by the static shift, and it resolves all of the model parameters accurately.

\section{Field example}

To demonstrate the efficacy of the proposed approach, a field example is presented from Singhbhum craton, eastern India. Singhbhum craton consists of a highly resistive and thick granitic layer that extends from the near surface to a great depth. Near-surface fractures in the granitic layer that allow groundwater movement through it affect the magnetotelluric data in this area. This groundwater movement produces electro-kinetic potential that modifies the electric field components. Figure 6 shows a classic field example in which the apparent resistivities in the transverse electric (TE) and transverse magnetic (TM) modes are widely different, and the phases in both of the polarization modes have similar magnitudes. This clearly indicates that the TE mode data are affected by the static shift. Dey [2005] estimated the resistivity as of the order of $70,000 \Omega \mathrm{m}$, with a thickness of $25 \mathrm{~km}$ to $30 \mathrm{~km}$ for the Singhbhum granite. These estimations are grossly inaccurate and overestimated. As discussed in the theoretical examples, in the absence of static-shift-corrected data, both the resistivity and the thicknesses are over estimated when the static-shift factor is greater than unity. It is important to note that for $S<1$, an opposite, underestimated, result would be obtained.

As the data indicate the 1-D structure up to large time periods, it is best to test the proposed approach. Field data up to $4096 \mathrm{~s}$ is presented in Figure 6; however, these data show multidimensionality after $800 \mathrm{~s}$. Therefore, to constrain the inversion, the data up to $800 \mathrm{~s}$ are used in the inversion. Furthermore, inversion should be attempted to model the minimum structures in the subsurface that fit the observed data very well. We systematically optimized the 3-, 4- and 5-layer models using joint inversion. It was always possible to obtain the correct fit with the apparent resistivity data and the estimation of the static shift of similar magnitude. However, the observed phase data did not fit well with the 3and 4-layer model data. Therefore, a 5-layer model was considered to match both the apparent resistivity data and the phase data, and the corresponding results are presented.

First, the 1-D joint inversion of the TE mode data (apparent resistivity and phase) is performed without
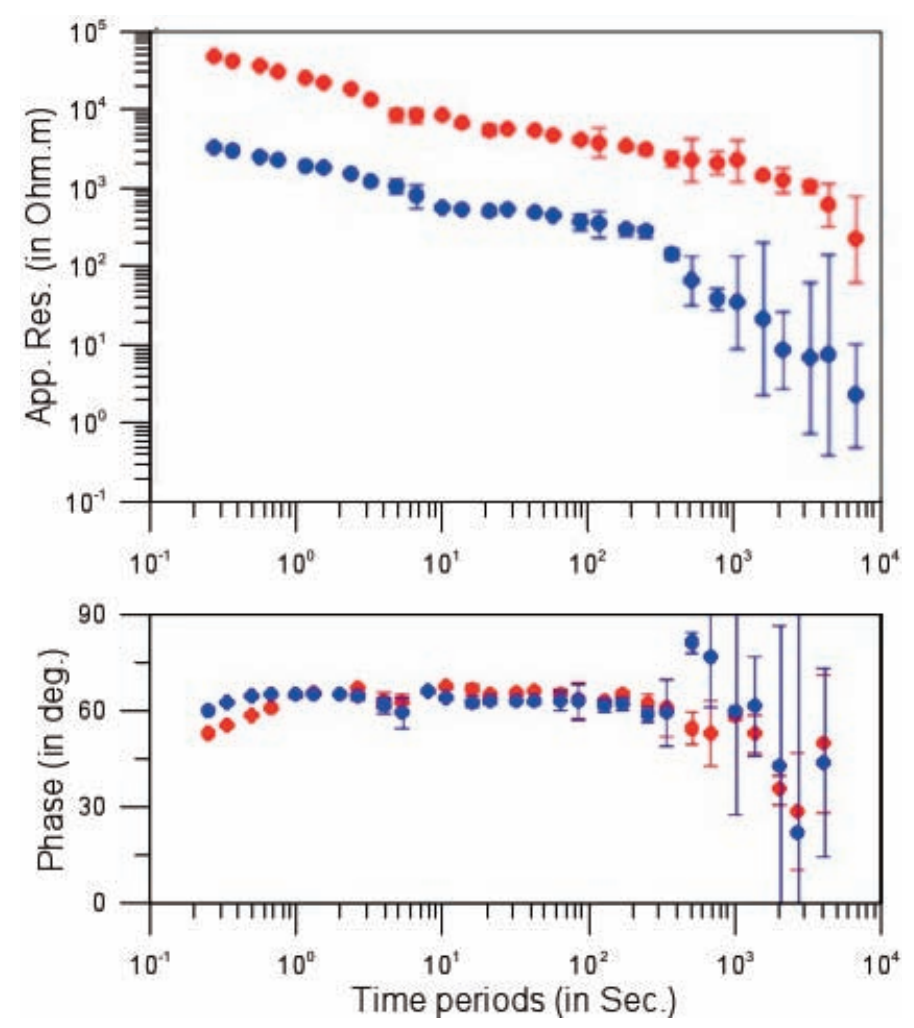

Figure 6. Classic field example showing the apparent resisitivity data (top) and the phase data (bottom) in transverse electric (red) and transverse magnetic (blue) modes with static shift [Dey 2005].

optimizing the static shift. It is important to note that a wider search range was required when the static shift was not optimized. Although the model data fit very well with the observed data (Figure 7), the resistivities and thicknesses are overestimated and geologically irrelevant (Table 8). Subsequently, the TE mode data are interpreted and the static shift is also optimized. The model response is modified according to the magnitude of the optimized static shift, which is $8.76 \pm 1.24$. Interestingly, the modified model data fit the observed data very well. Although the fit of the model data is similar, the delineated model parameters obtained after optimization of the static shift represent the actual subsurface structures (Table 8). As the fit is the same as that presented in Figure 7, similar plots are not repeated for brevity. Finally, the TM mode data are interpreted, and these results correlate very well with the TE mode interpretation that was also optimized for the static shift. Interestingly, the static shift parameter for the TM model is $1.03 \pm 0.01$, which suggests that these data are not affected by the static shift. The relation between the inverted model parameters for the TE data without and with optimization of the static shift follows a similar trend to that observed for the synthetic data for the various models. The interpreted results suggest that the thickness of the Singhbhum granite is about $14 \mathrm{~km}$. Two conducting layers with different resistivities are seen below the Singhbhum granite, which have a total thickness of about $15 \mathrm{~km}$. A resistive layer lies below this conducting 


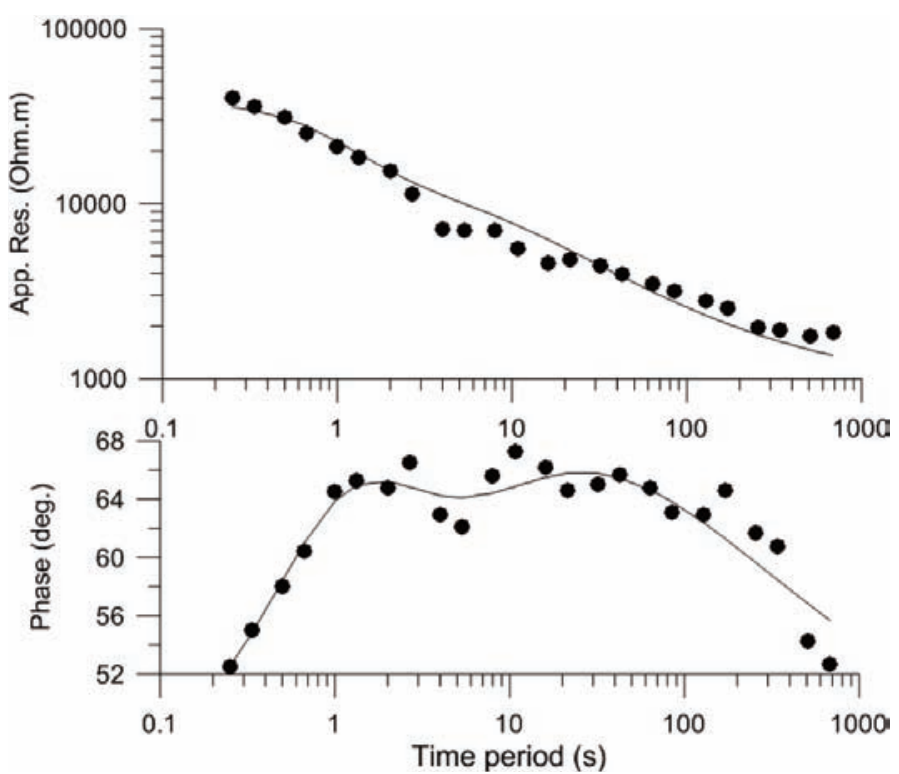

Figure 7. Fits for the apparent resisitivity data (top) and the phase data (bottom) for the observed (solid circles) and model (lines) responses for the transverse electric mode data.

strata, with a thickness of $7 \mathrm{~km}$ to $8 \mathrm{~km}$. The magnetotelluric data suggest that the heterogeneous structure lies below this resistive layer. This interpretation suggests that up to $36 \mathrm{~km}$ to $37 \mathrm{~km}$ depth of the structure is 1-D. This coincides well with the Moho depth in this region.

\section{Conclusions}

Magnetotelluric sounding is a very important geophysical method for investigation of subsurface structures. The measured apparent resistivity data are often affected by static shift. The static shift should be removed before data interpretation, or it should be optimized together with the other model parameters simultaneously, for accurate estimation of the subsurface structures. The global optimization method is presented for the interpretation of 1-D magnetotelluric sounding data, as well as for the optimization of the static shift. It can be concluded that the individual inversion of either the apparent resistivity data or the phase data without or with optimization of the static shift is not reliable. Even the joint inversion of the magnetotelluric sounding data without taking static shift into consideration leads to erroneous interpretations and yields inaccurate estimations of the model parameters. The estimated resistivities are multiplied by a factor equal to the amount of static shift, and the estimated thicknesses are multiplied by factor equal to the square root of the amount of static shift, with respect to the true resistivities and thicknesses, respectively. However, joint inversion of the apparent resistivity and phase data by also taking the static shift as one of the model parameter in the optimization yields very good estimations of all of the parameters, including the static shift. The accuracy of this approach is shown by taking a number of models that deal with synthetic and noisy data. This approach can also be used when the data are not affected by static shift. Joint inversion in such cases yields a static shift very close to unity. The efficacy of this approach is demonstrated with a classic field example from Singhbhum granitic craton, eastern India, where the TE data were affected by static shift. The inverted model parameters without and with static-shift optimization are in very good agreement with the theoretical results. The

\begin{tabular}{|c|c|c|c|c|c|}
\hline Parameters & Search range & $\begin{array}{c}\text { Without } \\
\text { optimizing static } \\
\text { shift (TE) }\end{array}$ & Search range & $\begin{array}{c}\text { With } \\
\text { optimizing } \\
\text { static shift (TE) }\end{array}$ & $\begin{array}{l}\text { With optimizing } \\
\text { static shift (TM) }\end{array}$ \\
\hline$\rho_{1}(\mathbf{\Omega m})$ & $10000-100000$ & $33293 \pm 1620$ & $1000-10000$ & $3735 \pm 391$ & $3134 \pm 540$ \\
\hline $\boldsymbol{\rho}_{2}(\mathbf{\Omega} \mathbf{m})$ & $1000-10000$ & $5547 \pm 810$ & $100-1000$ & $486 \pm 53$ & $283 \pm 28$ \\
\hline $\boldsymbol{\rho}_{3}(\boldsymbol{\Omega m})$ & $1000-10000$ & $2093 \pm 171$ & $100-1000$ & $239 \pm 28$ & $76 \pm 14$ \\
\hline $\boldsymbol{\rho}_{4}(\mathbf{\Omega m})$ & $10000-100000$ & $76646 \pm 2012$ & $1000-10000$ & $6683 \pm 534$ & $8751 \pm 476$ \\
\hline $\mathbf{P}_{5}(\mathbf{\Omega m})$ & $100-1000$ & $830 \pm 42$ & $10-1000$ & $94 \pm 6$ & $47 \pm 4$ \\
\hline$h_{1}(m)$ & $5000-50000$ & $41064 \pm 3460$. & $2000-25000$ & $14743 \pm 2890$ & $12960 \pm 1586$ \\
\hline $\mathbf{h}_{2}(\mathbf{m})$ & $5000-50000$ & $23470 \pm 2056$ & $2000-25000$ & $8762 \pm 428$ & $9980 \pm 687$ \\
\hline $\mathbf{h}_{3}(\mathbf{m})$ & $5000-50000$ & $19521 \pm 4351$ & $2000-25000$ & $4795 \pm 619$ & $4841 \pm 364$ \\
\hline $\mathbf{h}_{4}(\mathbf{m})$ & $5000-50000$ & $17942 \pm 2970$ & $2000-25000$ & $6449 \pm 840$ & $8816 \pm 1045$ \\
\hline$S$ & - & - & $1-20$ & $8.76 \pm 1.24$ & $1.03 \pm 0.01$ \\
\hline Misfit error & - & $2.01 \times 10^{-3}$ & - & $2.03 \times 10^{-3}$ & $6.06 \times 10^{-4}$ \\
\hline
\end{tabular}

Table 8. Field data: inversion results without and with optimization of static shift. TE, transverse electric mode; TM, transverse magnetic mode. 
inverted model parameters without optimization of the static shift are found to be geologically irrelevant, even though the model data fit the field data extremely well. However, the model parameters obtained after optimization of the static field yield accurate results that are geologically relevant. The global optimization of the field TM data shows that when the data are not affected by static shift, then the static-shift parameters can also be optimized. Moreover, in such cases, the static-shift parameter will be close to unity. This means that the approach is more robust and versatile for the interpretation of magnetotelluric data, irrespective of whether they are affected by static shift. Hence, this can be used to obtain very accurate estimations of the resistivities and thicknesses of subsurface structures.

Acknowledgements. We would like to thank the Editor and Reviewers for comments and suggestions that helped us to improve the manuscript. This study is part of a project sponsored to by the DST, Goverment of India, as a FIST Programme to the Department of Geology and Geophysics, IIT, Kharagpur, India.

\section{References}

Bahr, K. (1991). Geological noise in magnetotelluric data: a classification of distortion types, Phys. Earth Planet. Int., 66, 24-38.

Beamish, D. and J.M. Travassos (1992). A study of static shift removal from magnetotelluric data, J. Appl. Geophys., 29, 157-178.

Berdichevsky, M.N., L.L. Vanyan, V.A. Kuznetsov, V.T. Levadny, M.M. Mandelbaum., G.P. Nechaeva, B.A. Okulessky, P.P. Shilovsky and I.P. Shpak (1980). Geoelectric model of the Baikal region, Phys. Earth Planet. Int., 22 (1), 1-11.

Cerv, V., J. Pek and M. Menvielle (2010). Bayesian approach to magnetotelluric tensor decomposition, Annals of Geophysics, 53 (2), 21-32; doi: 10.4401/ag-4681.

de Groot-Hedlin, C. (1991). Removal of static shift in two dimensions by regularized inversion (short note), Geophysics, 56, 2102-2106.

Dey, S. (2005). Magnetotelluric survey in the northern part of eastern Ghats Mobile Belt and the southern part of Singhbhum craton across the Archaean-Proterozoic contact, eastern India, Ph.D. Thesis, IIT Kharagpur, India.

Dosso, S.E. and D.W. Oldenburg (1991). Magnetotelluric appraisal using simulated annealing, Geophys. J. Int., 106, 370-385.

Groom, R.W. and R.C. Bailey (1989). Decomposition of magnetotelluric impedance tensors in the presence of local three-dimensional galvanic distortion, J. Geophys. Res., 94, 1913-1925.

Jones, A.G. (1988). Static shift of magnetotelluric data and its removal in a sedimentary basin environment, Geophysics, 53, 967-978.

Juan, L.F.M., G.G. Esperanza, P.F.Á. José, A.K. Heidi and O.M.P. César (2010). PSO: A powerful algorithm to solve geophysical inverse problems: application to a 1D-DC resis- tivity case, J. Appl. Geophys., 71, 13-25.

Larsen, J.C. (1977). Removal of local surface conductivity erects from low frequency mantle response curves, Acta Geodaet., Geophysics. Et Montanist. Acad. Sci. Hung., 12 (1-3), 183-186.

Macnae, J., L. Lay and L.Weston (1998). Measurement of static shift in MT and CSAMT surveys, Expl. Geophys., 29, 494-498.

Pellerin, L. and G.W. Hohmann (1990). Transient electromagnetic inversion: A remedy for magnetotelluric shifts, Geophysics, 55, 1242-1250.

Rothman, D.H. (1985). Nonlinear inversion, statistical mechanics and residual statics estimation, Geophysics, 50, 2784-2796.

Rothman, D.H. (1986). Automatic estimation of large residual statics correction, Geophysics, 51, 337-346.

Sen, M.K., B.B. Bhattacharya and P.L. Stoffa (1993). Nonlinear inversion of resistivity sounding data, Geophysics, 58, 496-507.

Sen, M.K. and P.L. Stoffa (1995). Global Optimization Methods in Geophysical Inversion, Elsevier, Amsterdam, 277 pp.

Sharma, S.P. and P. Kaikkonen (1998). Two-dimensional nonlinear inversion of VLF-R data using simulated annealing, Geophys. J. Int., 133, 649-668.

Sharma, S.P. and P. Kaikkonen (1999). Appraisal of equivalence and suppression problems in 1-D EM and DC measurements using global optimization and joint inversion, Geophysical Prospecting, 47, 219-249.

Sharma, S.P. and S.K. Verma (2003). Solutions of the inherent problem of the equivalence in direct current resistivity and electromagnetic methods through global optimization and joint inversion by successive refinement of model space, presented in 23rd General Assembly of the IUGG 2003 (30 June-11 July), Sapporo, Japan.

Sternberg, B.K., J.C. Washburne and L. Pellerin (1988). Correction for the static shift in magnetotellurics using transient electromagnetic soundings, Geophysics, 53, 1459-1468.

Swift, C.M. (1967). A magnetotelluric investigation of an electrical conductivity anomaly in the southwesterm United States, Ph.D. thesis, M.I.T., U.S.A.

Tarantola, A. (1987). Inverse Problem Theory, Methods of data Fitting and Model Parameter Estimation, Elsevier Publishing Company, 630 pp.

Tournerie, B., M. Chouteau and D. Marcotte (2007). Magnetotelluric static shift: Estimation and removal using the cokriging method, Geophysics, 72 (1), F25-F34.

Vanyan, L.L., I.V. Yegorov, P.P. Shilovsky, I.M. Al'Perovich, V.M. K'Nikiforov and O.V. Volkova (1983). Characteristics of deep electrical conductivity of Northern Sakhalin, Izvestiya, Earth Physics, 19, 208-214.

Vedanti, N., R. Srivastava, J. Sagode and V.P. Dimri, (2005). An efficient 1D Occam's inversion algorithm using analytically computed first- and second-order derivatives for 
DC resistivity soundings, Computers \& Geosciences, 31 , 319-328.

Vozoff, K. (1972). The magnetotelluric method in the exploration of sedimentary basins, Geophysics, 37, 98-141.

Vozoff, K. and D.L.B. Jupp (1975). Joint inversion of geophysical data, Geophys. J. Roy. Astr. Soc., 42, 977-991.

Wang, Q., D. Tan and Z. Yao (1997). Removal of static shift in MT data using TEM soundings, The Leading Edge, 16, 358-359.

Wannamaker, P.E., G.W. Hohmann and S.H. Ward (1984). Magnetotelluric responses of three-dimensional bodies in layered earths, Geophysics, 49, 1517-1533.

${ }^{\star}$ Corresponding author: Shashi Prakash Sharma, Indian Institute of Technology, Dep. of Geology and Geophysics, Kharagpur, India; email: spsharma@gg.iitkgp.ernet.in.

(C) 2011 by the Istituto Nazionale di Geofisica e Vulcanologia. All rights reserved. 